\title{
EGYPTIAN LICENSE PLATE RECOGNITION USING ENHANCED STROKE WIDTH TRANSFORMATION AND FUZZY ARTMAP
}

\author{
${ }^{1}$ Alaa Mohamed Youssef, ${ }^{2}$ Mohamed S. El-Mahallawy and ${ }^{3}$ Amr Badr \\ ${ }^{1}$ College of Computing and Information Technology, \\ ${ }^{2}$ College of Engineering and Technology, \\ Arab Academy for Science, Technology and Maritime Transport, Sheraton, Cairo, Egypt \\ ${ }^{3}$ Department of Computer Science, Faculty of Computers and Information, Cairo University, Giza, Egypt
}

Received 2013-12-23; Revised 2013-12-25; Accepted 2014-01-28

\begin{abstract}
License Plate Recognition (LPR) is the most important type of Intelligent Transportation System (ITS). LPR is used in many different types of ITS like electronic payment systems, toll station, parking fees, freeway and arterial management systems for traffic surveillance. Few years ago, Egyptians government changed the car license plate to include letters and numbers. So the needs for efficient LPR System for the new license plate are increased in different ITS fields. This study presents an enhanced LPR detection algorithm for the new Egyptian licenses plate. The detection enhancement is done using Stroke Width Transform algorithm to extract letters from candidate areas combined with Fuzzy ARTMAP classifier. Stroke Width Transform (SWT) is a state of art algorithm developed by Microsoft Research Lab for detecting text in natural scene, it seeks to find the value of stroke width for each image pixel and demonstrate its use on the task of text detection in natural images. This study is focusing on detecting Arabic letters in the candidate license plate area using SWT image map instead of binary image map where not all Arabic letters have uniformly stroke width and some letters have a dot above and below it. The proposed model shows $26 \%$ detection accuracy enhancement than conventional LPR systems (Sobel Edge detection with binary image map using template matching technique).
\end{abstract}

Keywords: Image Processing, License Plate Recognition (LPR), License Plate Segmentation, Stroke Width Transformation, Optical Character Recognition (OCR), Fuzzy ARTMAP, Template Matching

\section{INTRODUCTION}

LPR is kind of Intelligent Transport Systems (ITS) that implements computer vision and character recognition algorithms (Ktata et al., 2013); LPR has become one of the most important digital image processing systems to be used. Since every vehicle carries a unique license plate, LPR is used as core modules for intelligent infrastructure systems like (electronic payment systems, toll station, parking fees, freeway and arterial management systems for traffic surveillance (Anagnostopoulos et al., 2008; Maglad, 2012).

Corresponding Author: Alaa Mohamed Youssef, College of Computing and Information Technology, Arab Academy for Science, Technology and Maritime Transport, Sheraton, Cairo, Egypt
LPR system is effective in crowded cities where the controlling of vehicles becomes a big problem and difficult to solve. For example Cairo, The capital of Egypt, is a crowded city where the number of vehicles increased because of population growth and due to the cheapness of domestic cars and each family may have one or more car.

LPR models are composed of three processing steps: (1) License plate detection, (2) Character segmentation, (3) Character recognition. The first two steps incorporate image processing techniques on standalone images or frame sequences (Videos), whose evaluation relies on the true recognition rate and the error recognition rate. 
There are many systems of LPR; these systems can be classified as: Template matching method (Cowell, 1995) (Shapiro et al., 2006) and learning based method (Zheng et al., 2005) (Parisi et al., 1998). Most of the algorithms have limiting conditions (Emiris and Koulouriotis, 2001), such as fixed illumination (Davies et al., 1990), fixed type of license plate, stationary backgrounds (Salgado et al., 1999), prescribed driveways (Parisiet al., 1998) (Miyamoto et al., 1991), limited vehicle speeds (Adorni et al., 1998; Broumandnia and Fathi, 2005).

The Egyptian license plate features such as color, numbers and letter specification depend on the issue purpose and the Governorate of release. The license plate has a fixed size $17 \times 32 \mathrm{~cm}$ divided horizontally into two parts. The ratio between the upper colored side and the white part is 1:2 (Massoud, 2013).

Stroke Width Transformation (SWT) (Epshtein et al., 2010) is a novel state of art image operator for detecting text from natural scene; SWT converts the image from an array contains gray values to an array contains likely stroke widths for each pixel. This information suffices for extracting the text by measuring the width variance in each component since text tends to maintain fixed stroke width which puts it apart from other image elements such as foliage.

The proposed model used SWT (Epshtein et al., 2010) to extract objects with similar stroke width. The SWT processing for whole image consume long time, so Sobel edge detection is used to extract candidate areas to be processed by SWT which reduce processing time. Other morphological image analysis is used to detect the properties of each extracted stroke width region.

The rest of this study is organized as follows. Section II defines the problem statement. Section III discusses Stork Width Transformation algorithm. Section IV discusses the proposed model. Section V discusses experimental results. Section VI concludes the proposed model and results.

\section{PROBLEM STATEMENT}

SWT gave great advantage to extract text from natural scene by distinguishing the objects that have uniform stroke width. Recognition of license plate that contains Latin alphabetical is easier than Arabic alphabetical. As for the Arabic letters, a dot can distinguish between different letters. Many letters don't have uniform shape like " 9 " and when applying SWT on it, it will be detected as two strokes; the upper part as a cycle and the lower part as a letter which can be interfered with letter ", ". Also, if the license plate is dirty it may affect the stroke width. Egyptians license plate also contains elements that are not letters but have uniform stroke width like Arabic word "مصر" and "Egypt", so depending on SWT only is not enough. The proposed model overcomes the previous problems by keeping only strokes with predetermined black and white color properties otherwise the stroke is ignored.

\section{STROKE WIDTH TRANSFORMATION}

A typical stroke is defined as a contiguous part of an image that forms a band of a nearly constant width. The Stroke Width Transform is a local image operator which computes per pixel the width of the most likely stroke containing the pixel (Epshtein et al., 2010) which transforms the image data from containing color values per pixel to containing the minimum width of the most likely strokes width. The SWT operator is linear in the number of edge pixels in the image and also linear in the maximal stroke width, determined at the training stage. The output of the SWT is an image of size equal to the size of the input image where each element contains the width of the stroke associated with the pixel. SWT is defining a suitable image operator whose output enables fast and dependable detection of text. SWT is able to detect text regardless of its scale, direction, font and language. SWT do not assume to know the actual width of the stroke but rather recover it.

Figure 1a shows a typical stroke example on stroke pixels darker than the background pixels. The initial value of each element of the SWT is set to $\infty$. In order to recover strokes, SWT first compute edges in the image using canny edge detector (Canny, 1986). After that, a gradient direction $d_{p}$ of each edge pixel is considered as shown in Fig. 1b. If lies on a stroke boundary, then $d_{p}$ must be roughly perpendicular to the orientation of the stroke. SWT follow the ray $r=p+n . d_{p}, n>0$ until another edge pixel $\mathrm{q}$ is found. SWT consider then the gradient direction $d_{q}$ at pixel $q$. If $d_{q}$ is roughly opposite to $d p\left(d_{q}\right.$ $\left.=-d_{p} \pm I I / 6\right)$, each element $\mathrm{s}$ of the SWT output image corresponding to the pixels along the segment $[p, q]$ is assigned the width unless it already has a lower value as shown in Fig. 1c. Otherwise, if the matching pixel q is not found, or if $d_{q}$ is not opposite to $d_{p}$, the ray is discarded. Figure 1 shows the process of SWT computation. Figure 2 shows an example on how pixels are filled with the minimum width value. Figure $2 \mathbf{a}$ shows example on simple strokes fill, where the red pixel is filled with the minimum width between the L lengths of the vertical and horizontal rays passing through it. 
Alaa Mohamed Youssef et al. / Journal of Computer Science 10 (6): 961-969, 2014

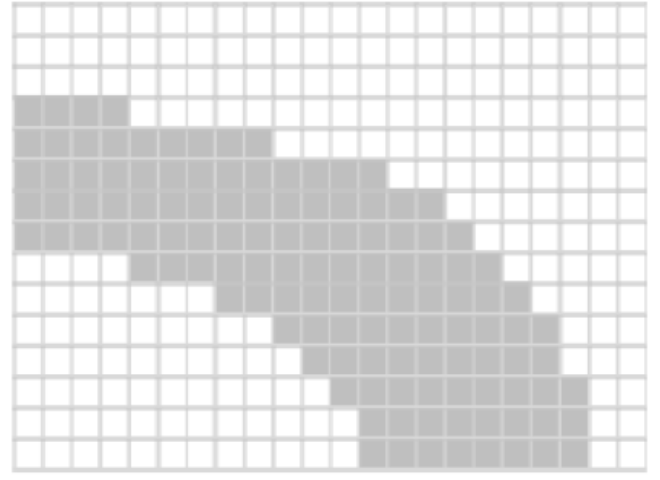

(a)

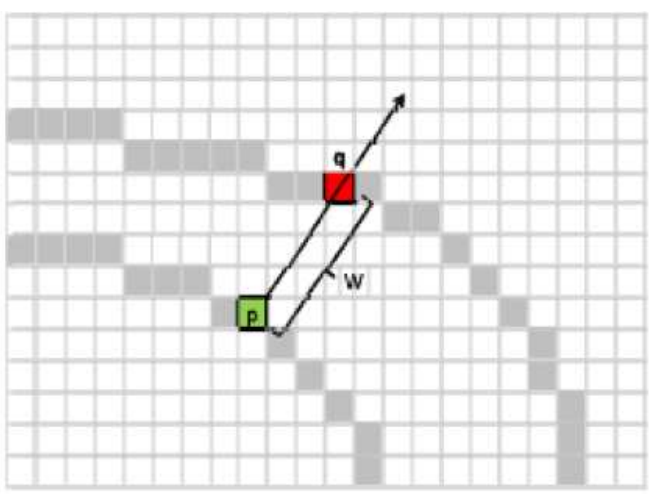

(b)

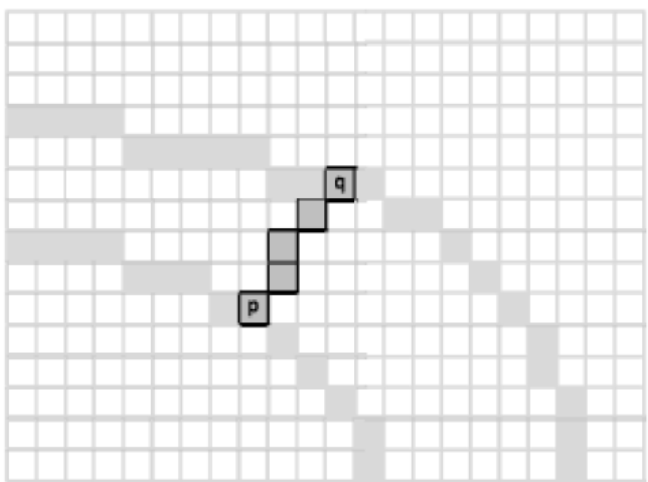

(c)

Fig. 1. Implementation of the SWT

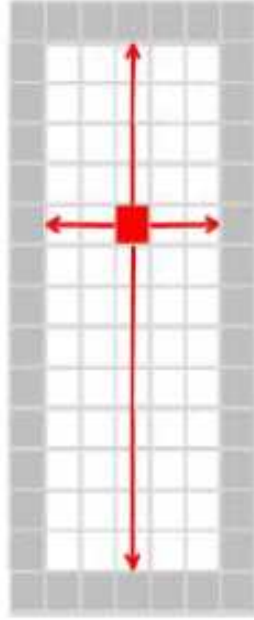

(a)

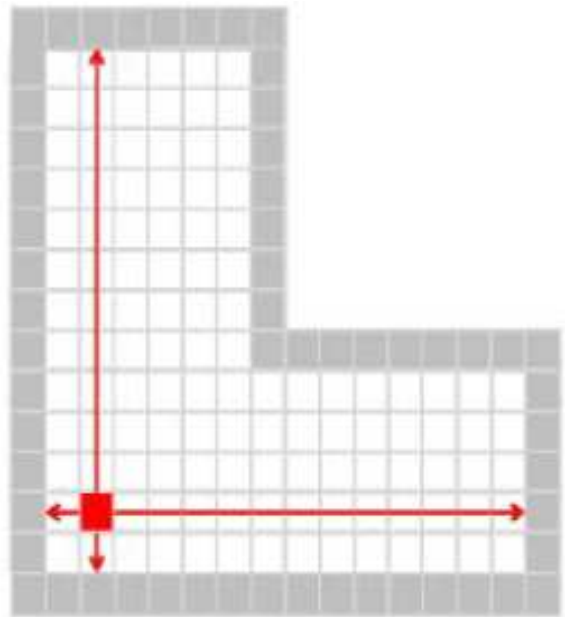

(b)

Fig. 2. Filling pixels with SWT values

Figure 2b shows an example on the SWT values in more complex situations, like corners, will not be true stroke widths after the first pass described above. Therefore, SWT pass along each non-discarded ray 
again and calculate the median SWT value $m$ of all its pixels and then set all the pixels of the ray with SWT values above $m$ to be equal to $m$.

\section{PROPOSED MODEL}

The proposed model consists of 3 stages: (1) Detect candidate areas, (2) Apply SWT on candidate areas and filter strokes and (3) Segmentation and character recognition. Figure 3 shows the structure of proposed model.

As a preprocessing step the input image is resized to 400 pixel height and the width is calculated dynamically to keep the aspect ratio of the image. By practical experiments, image resizing reduces processing time with the same results accuracy. Figure 4 shows resized image captured for a low speed moving car.

\subsection{Stage 1: Detect Candidate Areas}

This stage extracts the license plate candidate area using Sobel edge detection. Sobel is the most effective for LPR applications since Sobel method detect the outline of objects that have a clear contrast using two filters of size $3 \times 3$ pixels, one of which is designed to detect the horizontal difference and the other vertical difference. The filters have a double weight in the center of the order of 2 , which is more effective in practice (Fares, 2013). After applying Sobel edge detection as shown in Fig. 5, the morphological operations (Soille, 1999) dilation, filling closed areas and eroding takes place in sequence to extract candidate areas.

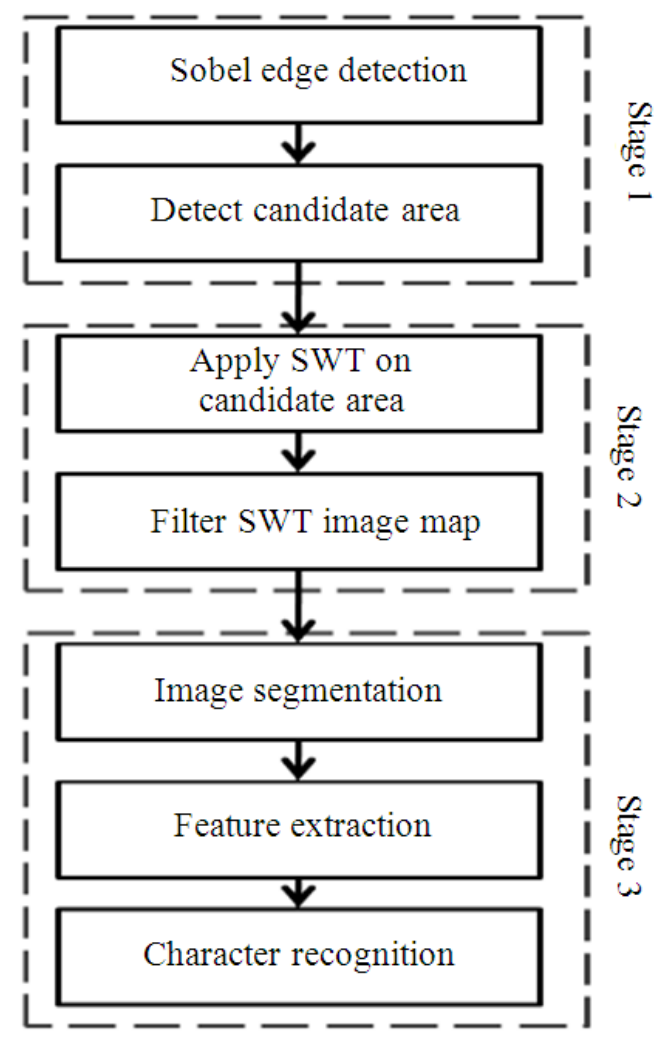

Fig. 3. Proposed model structure

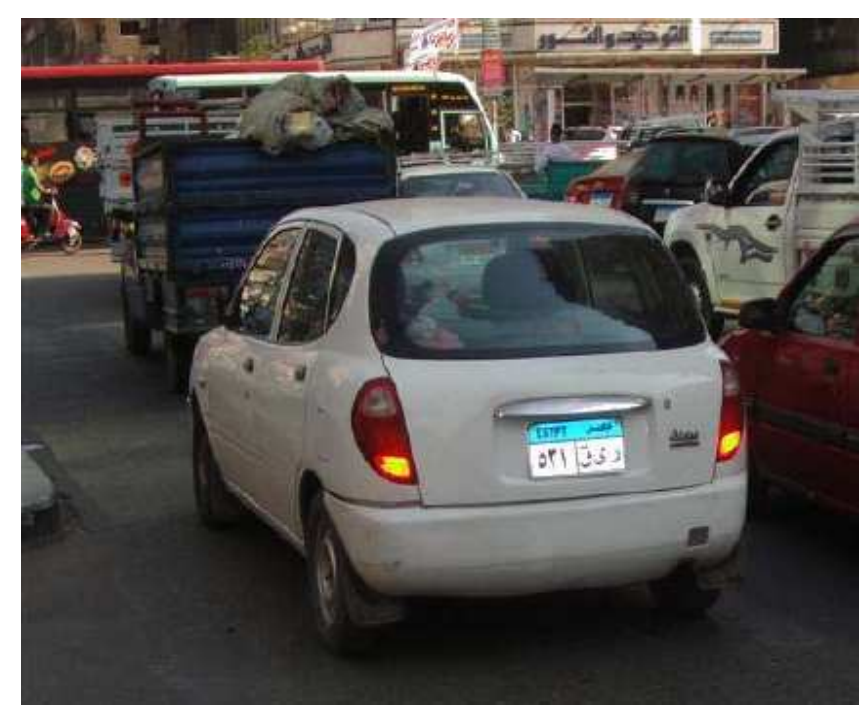

Fig. 4. The original image after resizing 


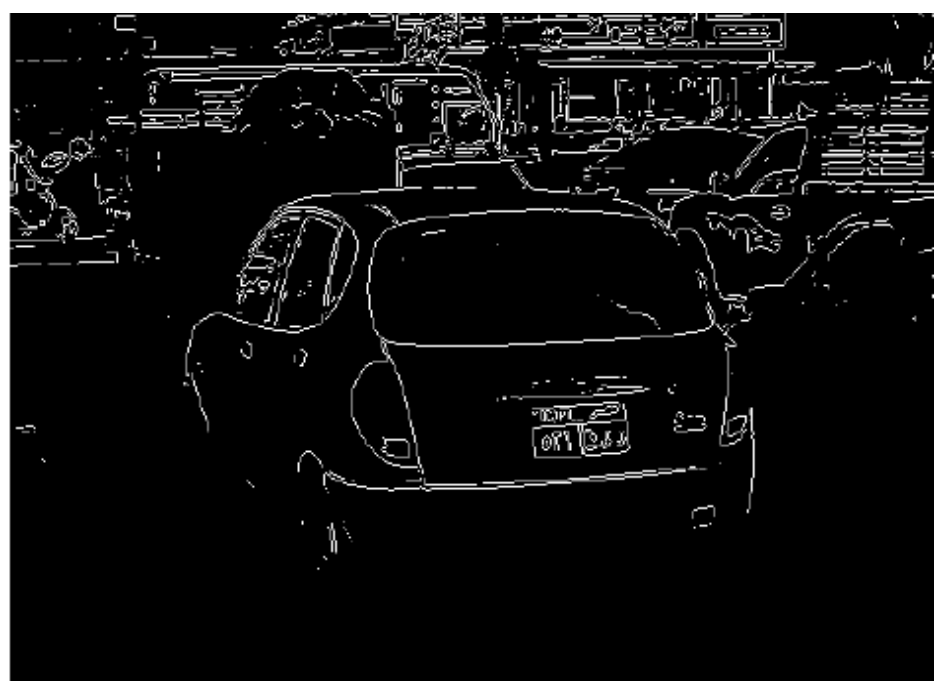

Fig. 5. Image after applying sobel edge detection

The result of previous operations gives the candidate parts that may contain license plate. Since the license plate dimensions are known and then each candidate area dimension is checked if it has area more than 2000 pixel and width/ height ratio between 2 and 9 (chosen empirically) or not as shown in Fig. 6. Otherwise the candidate areas are ignored, so it will decrease the number of the candidate areas to be processed by SWT and that will reduce the processing time.

\subsection{Stage 2: Apply SWT on Candidate Areas and Filter Strokes}

This stage takes the image inside each candidate regions in Fig. 6 and applies SWT on it. SWT will calculate the stroke width of every object inside the image. The result of this process is shown in Fig. 7.

The output from SWT algorithm may contain strokes which have uniform width but they are not letters in black and white colors. So each stroke will be checked that its color is black and its background is white based on threshold value chosen empirically. Based on this value, the stroke will be removed as shown in Fig. 8.

\subsection{Stage3: Segmentation and Character Recognition}

This stage is concerned with detecting character images from license plate image and matches its corresponding ASCII values. First a horizontal projection is applied on Fig. 8, Often the character part have the greater horizontal projection, so we will calculate each curve area and take the largest curve area and that will be the character area to be cropped as shown in Fig. 9 where the blue line shows the values of horizontal projection while the red line shows start and end points to be cropped from original image. The cropped image is shown in Fig. 10.

Vertical projection is then applied to separate each character as shown in Fig. 11. After detecting the start point and end point of each character, it is cropped into separated image and resized to $70 \times 50$, which is the size of letters trained by Fuzzy ARTMAP and then extracts its geometrical features (Ghosh, 2010) to be sent to Fuzzy ARTMAP to be classified and then its ASCII values returned.

Fuzzy ARTMAP is neural network architecture which is based on Adaptive Resonance Theory (ART) (Chralampidis et al., 2001). It has been used in condition monitoring. The Fuzzy ARTMAP architecture is capable of fast, online, supervised incremental learning, classification and prediction. The fuzzy ARTMAP operates by dividing the input space into a number of hyper boxes, which are mapped to an output space. Fuzzy ARTMAP is preferred due to its incremental learning ability. As new data is sampled, there will be no need to retrain the network.

The proposed model detected the license plate region correctly as shown in Fig. 12 where the green rectangle is the license plate region while the red rectangle shows non license plate region. 
Alaa Mohamed Youssef et al. / Journal of Computer Science 10 (6): 961-969, 2014

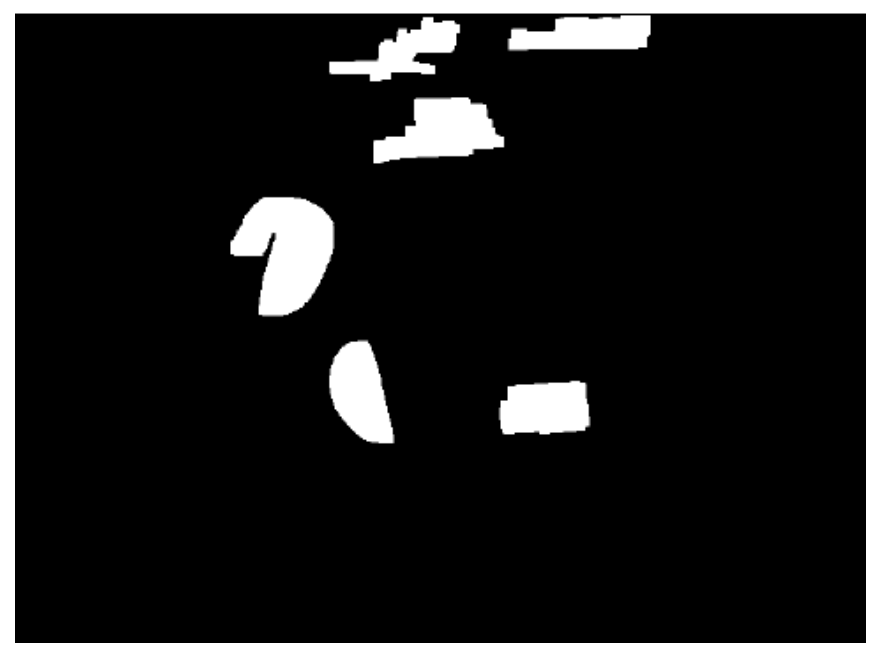

Fig. 6. Image after extracting candidate regions

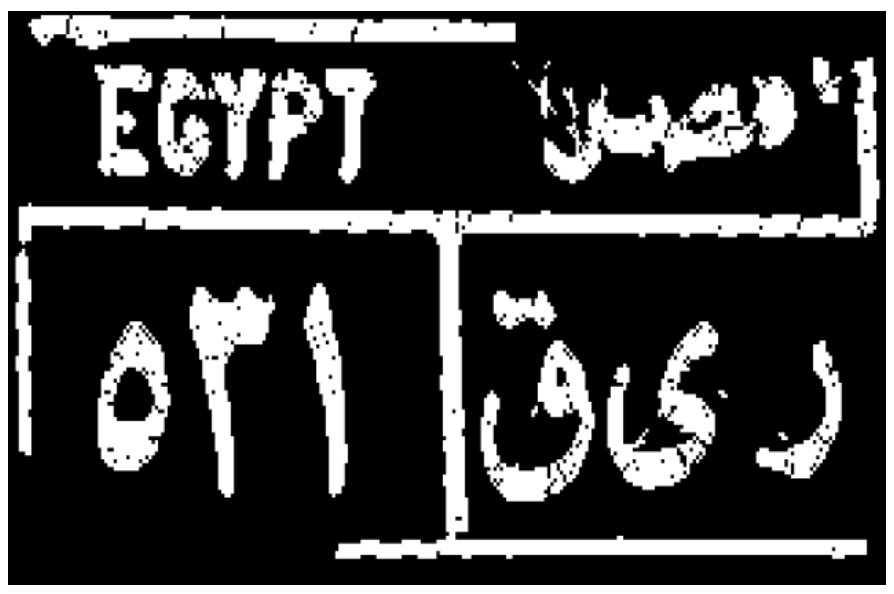

Fig. 7. SWT image for license plate region

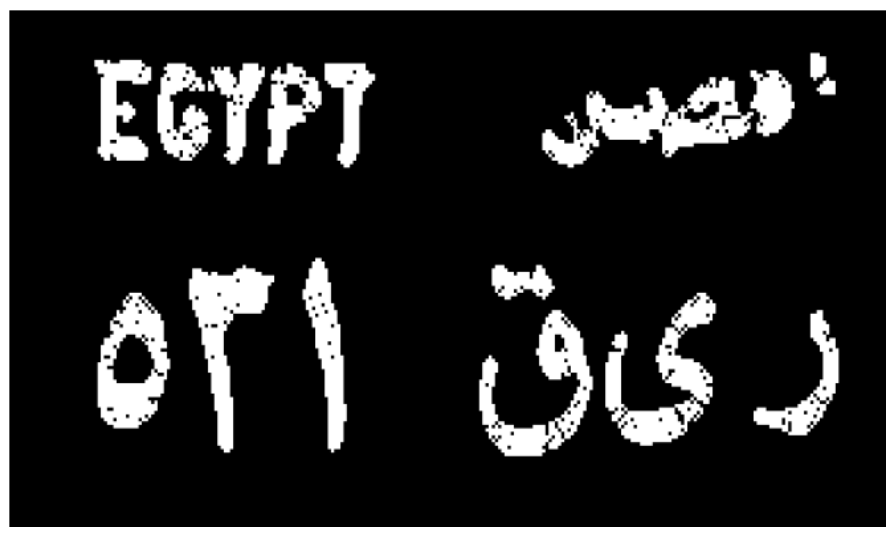

Fig. 8. SWT image after applying color filter 
Alaa Mohamed Youssef et al. / Journal of Computer Science 10 (6): 961-969, 2014

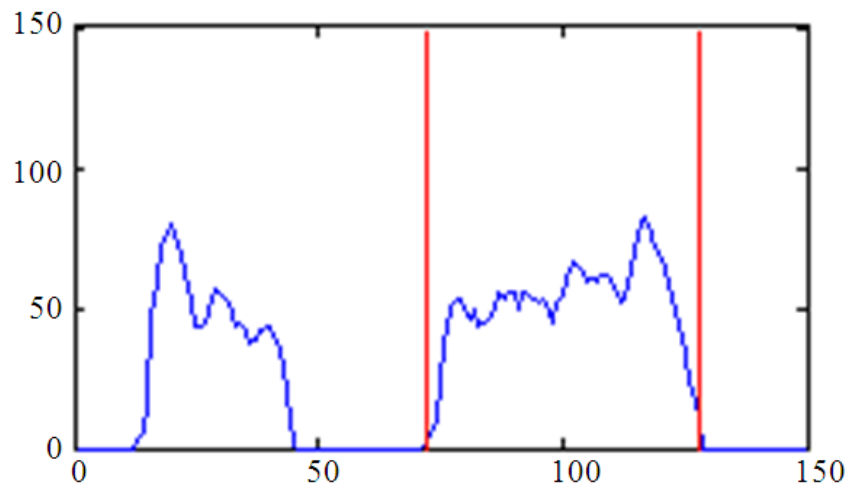

Fig. 9. SWT image horizontal projection

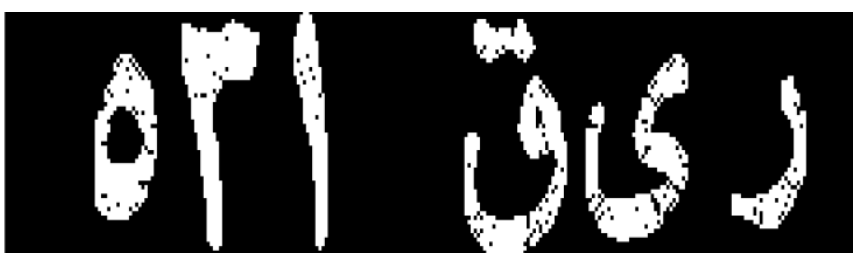

Fig. 10. SWT image after cropping

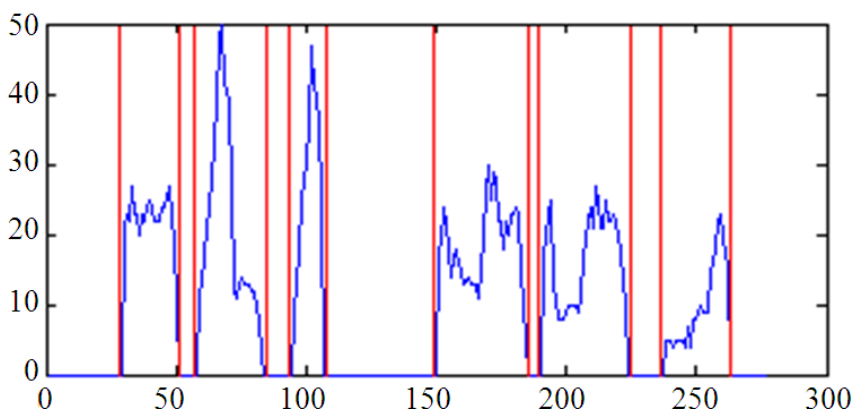

Fig. 11. SWT image vertical projections

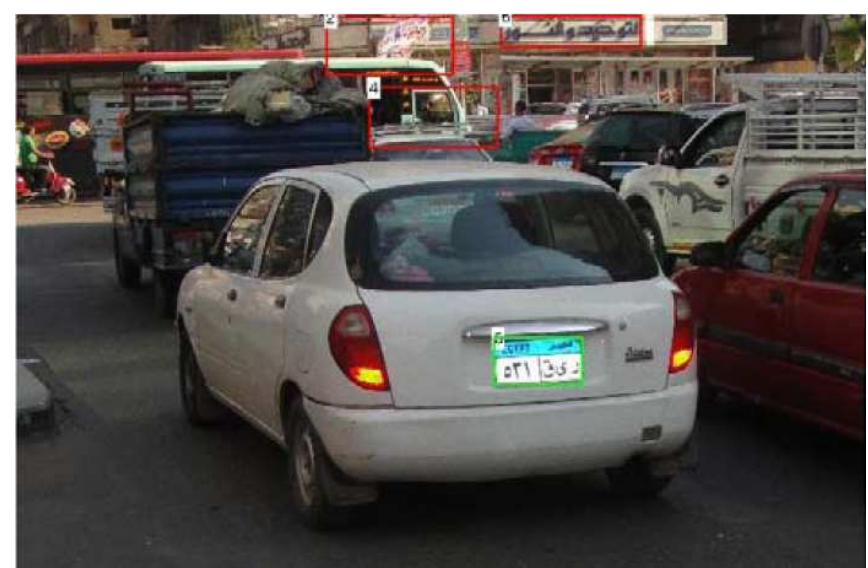

Fig. 12. Proposed model license plate region correctly detected 
Table 1. Experiment Results using template matching technique

\begin{tabular}{lll}
\hline Description & Conventional LPR system (\%) & Proposed Model (\%) \\
\hline Bad LPR detection & 10.6 & 5.6 \\
Correct license plate detection with character recognition error & 29.4 & 14.0 \\
Correct detection & 60 & 80.4 \\
\hline
\end{tabular}

Table 2. Experiment Results using Fuzzy ARTMAP classifier

\begin{tabular}{lll}
\hline Description & Conventional LPR system (\%) & Proposed model (\%) \\
\hline Bad LPR detection & 8.7 & 1.5 \\
Correct license plate detection with character recognition error & 24.5 & 12.5 \\
Correct detection & 66.8 & 86.0 \\
\hline
\end{tabular}

\section{EXPERIMENTAL RESULTS}

The proposed model is tested using 265 image datasets, the dataset contains images with different characteristic such as resolution, different cameras, day light time, night light time and captured for a moving and a static cars. The proposed model (Sobel Edge detection with SWT image map) performance is compared with a conventional LPR System for Egyptian license plate that was presented by (Massoud, 2013) where Sobel Edge detection with binary image map and using template matching technique was used. The proposed model and the conventional LPR (Massoud, 2013) are tested using two character recognition techniques, template matching and the Fuzzy ARTMAP classifier. The classifiers are trained using 722 Arabic letters images which are resized to $70 \times 50$.

The conventional LPR system using template matching technique detected $60 \%$ correctly with no character recognition errors, while the proposed model using template matching technique detected $80.4 \%$ correctly with no character recognition errors as shown in Table 1. The conventional LPR system using Fuzzy ARTMAP classifier detected $66.8 \%$ correctly with no character recognition errors, while the proposed model using Fuzzy ARTMAP classifier detected $86 \%$ correctly with no character recognition errors as shown in Table 2.

The previous tables show that the detection accuracy is improved using the proposed model compared to the conventional LPR (Massoud, 2013). This improvement is gained by using SWT which emphases letter strokes and ignores noise strokes and by employing Fuzzy ARTMAP instead of template matching to achieve better character recognition accuracy. The best correct detection results gained when we used SWT combined with Fuzzy ARTMAP classifier.

\section{CONCLUSION}

This study present an adaptation to a state of art Stroke Width Transformation technique for detecting Arabic letters for license plate recognition systems. SWT have great advantage for detecting text in natural scene, but it consume long time for large image, the proposed model extracted candidate area using Sobel edge detection and morphological operations, which decrease processing time than SWT only. The proposed model checks black and white color features for each stroke which eliminates non letter strokes and merge two strokes if they belong to the same letter. Two techniques are tested for character recognition, template matching technique and Fuzzy ARTMAP classifier. The proposed model using Fuzzy ARTMAP classifier shows $26 \%$ detection accuracy enhancement than using conventional LPR system (Sobel Edge detection with binary image map using template matching). The Fuzzy ARTMAP classifier used outperforms the template matching technique in detecting the Arabic characters. However, SWT has a limitation when dealing with high blurred image. Hence, images of high speed cars may not be detected correctly if it was taken by modest camera. So enhancing SWT performance for blurred image is a big challenge that has to be tackled in the future.

\section{REFERENCES}

Adorni, G., F. Bergent and S. Cagnoni, 1998. Vehicle license plate recognition by means of cellular automata. IEEE International Conference Intelligent Vehicles, (CIV' 98), IEEE Service Center, Piscataway NJ., pp: 689-693.

Anagnostopoulos, C.N.E., I.E. Anagnostopoulos, I.D. Psoroulas, V. Loumos and E. Kayafas et al., 2008. License plate recognition from still images and video sequences: A survey. IEEE Trans. Intell. Transp. Sys., pp: 377-391. DOI: 10.1109/TITS.2008.922938

Broumandnia, A. and M. Fathi, 2005. Application of pattern recognition for Farsi license plate recognition. Int. J. Graph. Vision Image Process., 5: 25-31. 
Canny, J. 1986. A computational approach to edge detection. IEEE Trans. Patt. Analysis Mach. Intell., 8: 679-714. DOI: 10.1109/TPAMI.1986.4767851

Chralampidis, D., T. Kasparis and M. Georgiopoulos, 2001. Classification of noisy signals using fuzzy artmap neural networks. IEEE Trans. Neural Netw., 15: 1023-1036. DOI: 10.1109/72.950132

Cowell, J. 1995. Syntactic pattern recognizer for vehicle identification numbers. Image Vision Comput., 13: 13-19. DOi: 10.1016/0262-8856(95)91464-O

Davies, P., N. Emmott and N. Ayland, 1990. License plate recognition technology for toll violation enforcement. Proceedings of the IEE Colloquium on Image Analysis for Transport Applications, Feb. 16-16, IEEE Xplore Press, London, pp: 1-5.

Emiris, D.M. and D.E. Koulouriotis, 2001. Automated optic recognition of alphanumeric content in car license plates in a semi-structured environment. Proceedings of the International Conference Image Processing, Oct. 7-10, IEEE Xplore Press, Thessaloniki, pp: 50-53. DOI: 10.1109/ICIP.2001.958048

Epshtein, B., E. Ofek and Y. Wexler, 2010. Detecting text in natural scenes with stroke width transform. Proceedings of the IEEE Conference on Computer Vision and Pattern Recognition, Jun. 13-18, IEEE Xplore Press, San Francisco, CA., pp: 2963-2970. DOI: 10.1109/CVPR.2010.5540041

Fares, C., 2013. Intelligent license plate recognition. Inter. J. New Comp. Architectures Applic., 3: 5469.

Ghosh, S., 2010. Offline handwritten digit recognition system. MSc Thesis, Jadavpur University, Kolkata, India.

Ktata, S., F. Benzarti and H. Amiri, 2013. License plate localization using Gabor filters and neural networks. J. Comput. Sci., 1341-1347. DOI: 10.3844/jcssp.2013.1341.1347
Maglad, K.W., 2012. A vehicle license plate detection and recognition system. J. Comput. Sci., 310-315. DOI: 10.3844 jessp.2012.310.315

Massoud, M., 2013. Automated new license plate recognition in Egypt. Alexandria Eng. J., 52: 319326. DOI: 10.1016/j.aej.2013.02.005

Miyamoto, K., K. Nagano, M. Tamagawa, I. Fujita and M. Yamamoto, 1991. Vehicle license-plate recognition by image analysis. Proceedings of the International Conference Industrial Electronics, Control Instrumentation, Oct. 28-Nov. 1, IEEE Xplore Press, Kobe, pp: 1734-1738. DOI: 10.1109/IECON.1991.239253

Parisi, R., E.D. Claudio, G. Lucarelli and G. Orlandi, 1998. Car plate recognition by neural networks and image processing. Proceedings of the IEEE International Symposium on Circuits and Systems, May 31-Jun. 3, IEEE Xplore Press, Monterey, CA., pp: 195-198. DOI: 10.1109/ISCAS.1998.703970

Salgado, L., J.M. Menendez, E. Rendon and N. Garcia, 1999. Automatic car plate detection and recognition through intelligent vision engineering. Proceedings of the IEEE 33rd Annual International Carnahan Conference on Security Technology, Oct. 05-07, IEEE Xplore Press, Madrid, pp: 71-76. DOI: 10.1109/CCST.1999.797895

Shapiro, V., G. Gluhchev and D. Dimov, 2006. Towards a multinational car license plate recognition system. Machine Vision Applic., 17: 173-183. DOI: 10.1007/s00138-006-0023-5

Soille, P., 1999. Morphological Image Analysis: Principles and Applications. 1st Edn., Springer, Berlin, ISBN-10: 3540656715, pp: 316.

Zheng, L., X. He, Q. Wu and T. Hintz, 2005. Character recognition of car number plates. Proceedings of the International Conference on Computer Vision, (CCV' 05), pp: 33-39. 